\title{
Criticality of the Higgs mass for the long-range quantum $X Y$ chain: Amplitude ratio between the Higgs and paramagnetic gaps
}

\author{
Yoshihiro Nishiyama \\ Department of Physics, Faculty of Science, Okayama University, Okayama 700-8530, \\ Japan
}

\begin{abstract}
The quantum $X Y$ spin chain with the interactions decaying as a power law $1 / r^{1+\sigma}$ of the distance between spins $r$ was investigated with the exact diagonalization method. Here, the constituent spin is set to $S=1$, which enables us to incorporate the biquadratic interactions so as to realize the order-disorder transition with the $\mathrm{O}(2)$ symmetry maintained. Thereby, in the ordered phase, we resolved the Higgs mass $m_{H}$ out of the Goldstoneexcitation continuum by specifying Higgs-particle's quantum numbers to adequate indices. We then turn to the analysis of the critical amplitude ratio $m_{H} / \Delta$ ( $\Delta$ : paramagnetic gap in the disordered phase). As the power of the algebraic decay $\sigma$ increases, the amplitude ratio $m_{H} / \Delta$ gets enhanced gradually in agreement with the $\epsilon(=4-D)$-expansion-renormalization-group result; here, we resort to the $\sigma \leftrightarrow D$ relation advocated recently in order to establish a relationship between the renormalization-group result and ours.
\end{abstract}

Keywords:

05.50.+q 05.10.-a 05.70.Jk 64.60.-i

\section{Introduction}

The spin systems with the long-range interactions have been investigated both theoretically $[1,2,3,4,5,6,7,8,9,10,11,12,13,14,15,16]$ and experimentally $[17,18,19,20,21,22]$. A main concern is to clarify how the criticality between the ordered and disordered phases is affected by the power of the algebraic decay $[7,8,9]$. Meanwhile, the idea was extended to the case of the quantum spin models [23, 24, 25, 26, 27, 28, 29, 30, 31, 32, 
33, 34]. In Fig. 1, we present the criticality chart [25, 30, 31, 32, 33, 34] for the quantum $X Y$ spin chain with the interactions decaying as a power law $1 / r^{1+\sigma}$ of the distance between spins $r$. For $\sigma \gtrsim 2$, the long-range interaction becomes irrelevant, and the criticality falls into the universality class of the classical two-dimensional $(D=2) X Y$ model (with the shortrange interactions). On the contrary, for $\sigma<2 / 3$, the criticality belongs to the mean-field type, namely, the $D=4$ universality class. In the intermediate regime $2 / 3 \leq \sigma \lesssim 2$, the power $\sigma$ interpolates both limiting cases, $D=2$ and 4 , smoothly. Actually, relying on the correspondence between the $\sigma$ - and $D$ mediated considerations, the authors of Ref. [13] analyzed the criticality for the $D=3$ (short-range) Ising model quantitatively. Experimentally, systems with variable-range interactions have been synthesized $[18,19,20]$. In this sense, the $\sigma \leftrightarrow D$ relation [8] is not a mere theoretical concept.

The criticality chart, Fig. 1, resembles to that of the classical counterpart $[1,2,7]$. In this paper, aiming to elucidate the quantum nature of this problem, we devote ourselves to the spectrum, namely, the Higgs (paramagnetic) excitation gap, $m_{H}(\Delta)$, in the (dis)ordered phase. Particularly, we estimate the critical amplitude ratio

$$
m_{H} / \Delta=\left.\frac{m_{H}(J)}{\Delta\left(2 J_{c}-J\right)}\right|_{J \rightarrow J_{c}^{+}},
$$

with the $X Y$ interaction $J$ and the power $\sigma$ ranging within $2 / 3<\sigma<2$. In Fig. 2, we present a schematic drawing for the spectrum in the disordered $\left(J<J_{c}\right)$ and ordered $\left(J>J_{c}\right)$ phases. The paramagnetic gap opens in the disordered phase, whereas in the ordered phase, the Higgs branch $m_{H}$ is embedded within the Goldstone-excitation continuum. Meanwhile, the amplitude ratio $m_{H} / \Delta$ was estimated for the generic values of $D$ by means of the $\epsilon$-expansion method [35]. For the fixed- $D=3$ systems, a good deal of analyses have been made [36, 37, 38, 39, 40,41]. As mentioned above, via the $\sigma \leftrightarrow D$ relation $[8,25]$, one is able to establish a relationship among these $D$ - and $\sigma$-mediated considerations.

We employed the exact diagonalization method, which enables us to calculate the energy gaps, $m_{H}$ and $\Delta$, directly by specifying the quantum numbers to Eqs. (3) and (4), respectively. So far, in the quantum-Monte-Carlo approach [36, 37, 38], the dynamical scalar susceptibility $\chi_{s}(\omega)[42,43]$ has been enumerated through the inverse Laplace transformation; see Appendix B of Ref. [37]. Because the disturbance $\chi_{s}(\omega)$ preserves the $\mathrm{O}(2)$ symmetry, 
it is less sensitive to the Goldstone excitations [44]; hence, a $m_{H}$ signal can be captured via $\chi_{s}(\omega)$ [43]. In the recent experiment for the ultra cold atom [45], essentially the same strategy is undertaken; for such boson system, the (dis)ordered phase should be interpreted as the superfluid (Mott insulator) phase. We stress that the exact diagonalization method admits $m_{H}$ and $\Delta$ without resorting to such elaborated techniques.

To be specific, we present the Hamiltonian for the one-dimensional quantum spin- $S=1 X Y$ model with the long-range interactions

$\mathcal{H}=-\frac{1}{\mathcal{N}} \sum_{i \neq j} J_{i j}\left(\frac{J}{2}\left(S_{i}^{+} S_{j}^{-}+S_{i}^{-} S_{j}^{+}\right)+\frac{J_{4}}{4}\left(\left(S_{i}^{+}\right)^{2}\left(S_{j}^{-}\right)^{2}+\left(S_{i}^{-}\right)^{2}\left(S_{j}^{+}\right)^{2}\right)\right)+D_{s} \sum_{i=1}^{N}\left(S_{i}^{z}\right)^{2}$.

Here, the quantum spin- $S=1$ operator $S_{i}^{ \pm, z}$ is placed at each one-dimensionallattice point, $i=1,2, \ldots, N$. The summation $\sum_{i \neq j}$ runs over all possible pairs $1 \leq i, j \leq N$, and the long-range interaction $J_{i j}$ decays as a power law, $J_{i j}=1 / \sin (\pi|i-j| / N)^{1+\sigma}$, with the variable exponent $\sigma$. Here, we implemented the periodic-boundary condition for the spin chain; what is meant by the expression for $J_{i j}$ is that the distance between the spins, $i$ and $j$, is given by the chord length, $\sin (\pi|i-j| / N)$, rather than the arc, $|i-j|$. The $X Y$ interaction $J$ induces the order-disorder transition (see Fig. 2), whereas the biquadratic interaction $J_{4}=1[46,47,48]$ is fixed. The single ion anisotropy $D_{s}=0.1(2-\sigma)$ is included so as to improve the finite-size behaviors [49]; the single ion anisotropy drives the $X Y$ phase to the massive phase $[50,51,52]$ in $D=2$ dimensions $(\sigma=2)$, and we incorporated the diminution factor $(2-\sigma)$. The normalization (Kac) factor $\mathcal{N}[53,54]$ is given by $\mathcal{N}=N^{-1} \sum_{i \neq j} \sin (\pi|i-j| / N)^{-1-\sigma}$.

The rest of this paper is organized as follows. In the next section, we show the numerical results. A brief account of the simulation algorithm is given as well. In the last section, we address the summary and discussions.

\section{Numerical results}

In this section, we present the numerical results. We employed the exact diagonalization method for the Hamiltonian (2) with $N \leq 22$ spins. Before commencing detailed scaling analyses for $m_{H}$ and $\Delta$, we give a brief account of the numerical algorithm. The Hamiltonian has a number of symmetries, i.e., good quantum numbers, with which we are able to diagonalize the Hamiltonian within the restricted Hilbert space. The quantum-number 
specification scheme for $m_{H}$ and $\Delta$ is as follows: The numerical diagonalization was performed within the zero-momentum subspace $k=0$, at which the elementary-excitation gap opens; namely, both the ground- and first-excitedstate levels locate. We further specify the subspace with an additional quantum number $S_{\text {tot }}^{z}$, which corresponds to the total magnetization operator, $\sum_{i=1}^{N} S_{i}^{z}$; this quantum number reflects the $\mathrm{O}(2)$ symmetry of $\mathcal{H}$. Within the restricted Hilbert space specified by $k=0$ and $S_{t o t}^{z}$, the Higgs mass is given by

$$
m_{H}=E_{1}(0)-E_{0}(0)
$$

with the ground- (first excited-) state energy $E_{0(1)}\left(S_{\text {tot }}^{z}\right)$ within the sector $S_{\text {tot }}^{z}$. The paramagnetic excitation belongs to the $S_{\text {tot }}^{z}= \pm 1$ sector, and hence, the gap is given by the formula

$$
\Delta=E_{0}(1)-E_{0}(0) .
$$

This $\Delta$ branch becomes the Goldstone mode in the adjacent phase (ordered phase). Note that the $m_{H}$ mode exists far above the collection of the Goldstone excitations (continuum), and such level specification is significant to resolve the former out of the latter.

\subsection{Scaling analysis of the dynamical critical exponent z: A preliminary survey}

In this section, based on the scaling theory developed in Ref. [25], we analyze the Higgs mass $m_{H}$, Eq. (3). As a byproduct, we obtain the dynamical critical exponent $z$, which characterizes the anisotropy between the real-space and imaginary-time directions quantitatively.

In Fig. 3, we present the scaling plot, $\left(J-J_{c}\right) N^{1 / \nu}-N^{z} m_{H}$, for the fixed $\sigma=1.2$ and various system sizes, $(+) N=18,(\times) 20$, and $(*) 22$. The scaling parameters, namely, the critical point $J_{c}=0.30785$, the reciprocal correlation-length critical exponent $1 / \nu=0.636$, and the dynamical critical exponent $z=0.594$, were determined as follows. The critical point $J_{c}=$ $0.30785(40)$ was obtained through the least-squares fit for the $1 / N-J_{c}(N)$ data with $N=16,18, \ldots, 22$; here, the approximate critical point $J_{c}(N)$ is defined by

$$
\left.\partial_{J} m_{H}\right|_{J=J_{c}(N)}=0,
$$

for each $N$. Likewise, the dynamical critical exponent $z=0.594(1)$ was obtained via the least-squares fit for the $\left(\frac{N+(N+2)}{2}\right)^{-1}-z(N, N+2)$ data with 
$N=14,16, \ldots, 20$. The approximate dynamical critical exponent $z\left(N, N^{\prime}\right)$ is given by the logarithmic derivative of the finite-size Higgs mass

$$
z\left(N, N^{\prime}\right)=-\frac{\left.\ln m_{H}(N)\right|_{J_{c}(N)}-\left.\ln m_{H}\left(N^{\prime}\right)\right|_{J_{c}\left(N^{\prime}\right)}}{\ln N-\ln N^{\prime}}
$$

with a pair of system sizes $\left(N, N^{\prime}\right)$. Finally, the reciprocal correlation-length critical exponent $1 / \nu=0.636$ was calculated from the above-mentioned result $z=0.594$ with the aide of $1 / z \nu=1.07$ taken from Fig. 3a of Ref. [25].

The scaled data in Fig. 3 seem to obey the finite-size scaling satisfactorily. The Higgs mass appears to open in the ordered phase $J>J_{c}$. The criticality for $m_{H}$ is analyzed in depth afterwards. Noticeably enough, the $m_{H}$ branch opens also in the adjacent (disordered) phase $J<J_{c}$. This massive mode may correspond to the particle-hole-excitation threshold in the boson language $[37,38]$; further details as to the disordered phase are not pursued in this paper.

Similar analyses as that of Fig. 3 were carried out for various values of $\sigma$. The $\sigma$-dependent dynamical critical exponent $z$ is presented in Fig. 4. Here, as an error margin, we accepted the deviation between different extrapolation schemes, namely, $N^{-1}-z(N, N+2)$ and $N^{-2}-z(N, N+2)$ approaches, aiming to appreciate possible systematic errors other than the least-squares-fit error. The series of results in Fig. 4 indicate that the dynamical critical exponent $z$ reflects the variation of $\sigma$ sensitively. As a reference, in Fig. 4, we also present an approximate formula (dots) [25]

$$
z=\sigma / 2
$$

which is validated in the small- $\sigma$ side. Our data appear to obey the formula (7) for a considerably wide range of $\sigma$. However, around the upper and lower critical thresholds, $\sigma=2$ and $2 / 3$, respectively, there emerge systematic deviations, which may be attributed to the notorious logarithmic corrections to finite-size scaling $[3,10,11,26]$. Note that these thresholds correspond to the lower and upper critical dimensions, $D=2$ and 4 , respectively (see Eq. (11)), and inevitably, the finite-size scaling is affected by the marginal operators at these thresholds.

We address a number of remarks. First, the dynamical critical exponent $z$ is peculiar to the quantum criticality. The conventional short-range spin models exhibit $z=1$, namely, the restoration of the symmetry between the real-space and imaginary-time subspaces. On the contrary, as shown above, 
the long-range counterpart does exhibit the anisotropy, $z \neq 1$ [25], between these subspaces. In the quantum Monte Carlo simulation, a special care has to be paid so as to remedy this anisotropy in order to manage the finitesize-scaling properly. In the exact diagonalization approach, one is able to concentrate on the real-space sector, because the imaginary-time system size is infinite $\beta(=1 / T) \rightarrow \infty$ a priori; note that one can access the ground state $T=0$ directly. Recently, as for the quantum long-range systems, a variety of approaches such as the exact diagonalization method [53], the variational matrix product state with the quasi-particle ansatz [54], and the density-matrix renormalization group under the open boundary condition [55], have been utilized successfully. Because in the present analysis, the mass gaps right at the zone center $k=0$ are required, we resort to the exact diagonalization method under the periodic boundary condition. Last, in Fig. 3 , the minimum point for the Higgs gap seemingly locates slightly out of the critical point $\left(J-J_{c}\right) N^{1 / \nu} \approx 0.1$. However, in the thermodynamic limit, the deviation from the critical point $J_{c}$ vanishes as $J-J_{c} \approx 0.1 / N^{1 / \nu} \rightarrow 0$.

\subsection{Critical amplitude ratio $m_{H} / \Delta$}

Based on the finite-size-scaling analysis examined in the preceding section, we turn to the analysis of the critical amplitude ratio $m_{H} / \Delta$. To this end, we investigate the scaling behavior of the paramagnetic gap $\Delta$, Eq. (4). In Fig. 5 , we present the scaling plot, $\left(J-J_{c}\right) N^{1 / \nu_{-}} N^{z} \Delta$, for $\sigma=1.2$ and various system sizes, $(+) N=18,(\times) 20$, and $(*) 22$. The scaling parameters, $J_{c}=0.30785,1 / \nu=0.636$, and $z=0.594$, are the same as those of Fig. 3 . The scaled data fall into the scaling curve satisfactorily. The paramagnetic gap $\Delta$ opens in the disordered phase $J<J_{c}$; see the schematic diagram, Fig. 2 , as well. The paramagnetic mode $\Delta$ in the disordered phase turns into the Goldstone excitation in the adjacent phase (ordered phase) $J>J_{c}$. Actually, in $J>J_{c}$, there is shown a closure of the $\Delta$ branch, which is smaller than the Higgs gap $m_{H}$ in Fig. 3. Such Goldstone continuum place an obstacle to observe $m_{H}$ clearly. In this paper, we pick up the $m_{H}$ mode directly by specifying the quantum numbers to Eq. (3) adequately.

We then consider the amplitude ratio $m_{H} / \Delta$. In Fig. 6, we present the scaling plot, $\left(J-J_{c}\right) N^{1 / \nu}-m_{H}(J) / \Delta\left(2 J_{c}-J\right)$, for $\sigma=1.2$ and various system sizes, $(+) N=18,(\times) 20$, and $(*) 22$. The scaling parameters, $J_{c}=0.30785$, and $1 / \nu=0.636$, are the same as those of Fig. 3. Again, the collapse of the scaled data seems to be satisfactory. In the ordered phase $J>J_{c}$, the amplitude ratio takes a plateau with the height $m_{H} / \Delta \approx 1.8$. Such a feature 
indicates that that the amplitude ratio $m_{H} / \Delta$ indeed takes a constant value in proximity to the critical point.

The plateau in Fig. 6 exhibits a shallow bottom, which provides a good indicator as to the amplitude ratio for each $N$. Aiming to extrapolate $m_{H} / \Delta$ systematically to the thermodynamic limit $N \rightarrow \infty$, we carried out the least-squares fit for the plot $N^{-1}-\frac{m_{H}}{\Delta}(N)$ with $N=16,18, \ldots, 22$; here the approximate amplitude ratio is given by

$$
\frac{m_{H}}{\Delta}(N)=\left.\frac{m_{H}(J)}{\Delta\left(2 J_{c}-J\right)}\right|_{J=\bar{J}(N)},
$$

at the bottom of the plateau $\bar{J}(N)$ satisfying the extremum condition

$$
\left.\frac{\partial\left(m_{H}(J) / \Delta\left(2 J_{c}-J\right)\right)}{\partial J}\right|_{J=\bar{J}(N)}=0
$$

for each $N$. We arrived at $m_{H} / \Delta=1.980(14)$ via the extrapolation scheme.

We carried out similar analyses as that of Fig. 6 for various values of $\sigma$. The $\sigma$-dependent amplitude ratio $m_{H} / \Delta$ is presented in Fig. 7. Here, as an error margin, we accepted the deviation between different extrapolation schemes, namely, $N^{-1}-\frac{m_{H}}{\Delta}(N)$ and $N^{-2}-\frac{m_{H}}{\Delta}(N)$ fittings, aiming to appreciate possible systematic errors other than a fitting error. Our data in Fig. 7 indicate that the critical amplitude ratio $m_{H} / \Delta$ varies monotonically, as the decay rate $\sigma$ changes. In other words, the low-energy spectrum reflects the long-range-interaction decay rate $\sigma$ rather sensitively.

We address a number of remarks. First, the solution for the amplitude ratio $m_{H} / \Delta$ does not exist for exceedingly large $\sigma \gtrsim 1.6$. That is, the Higgs particle becomes unstable for large $\sigma$, and the plateau as in Fig. 6 disappears (shrinks) eventually. The end point $\sigma \approx 1.6$ corresponds to $D \approx 2.25$ according to the $\sigma \leftrightarrow D$ relation (11); the dimensionality $D=2.25$ is about to reach the lower critical dimension $D=2$. Last, the excitation gaps, $m_{H}$ and $\Delta$, as well as the amplitude ratio $m_{H} / \Delta$ exhibit smooth variations throughout the intermediate regime $2 / 3<\sigma<2$. As for the Ising counterpart [53, 54], an anomaly (a dynamical phase transition) was observed around $\sigma \approx 1$.3. Correspondingly, the critical amplitude ratio $m_{2} / m_{1}\left(m_{i}: i\right.$-th excitation gap) displays a non-monotonic behavior [56]; actually, a minimum locates in the midst of the intermediate regime as to $m_{2} / m_{1}$. 
2.3. Comparison with the preceding results via the $\sigma \leftrightarrow D$ relation [8, 25]

According to the elaborated $\epsilon$-expansion analysis, the critical amplitude ratio is given by

$$
m_{H} / \Delta=\sqrt{2}+\sqrt{2} \epsilon\left(\frac{4 \ln 2+3 \sqrt{3} \pi}{40}-\frac{1}{4}\right),
$$

with $D=4-\epsilon$. As a reference, in Fig. 7, we present the $\epsilon$-expansion result (dots). Here, we made use of the $\sigma \leftrightarrow D$ relation $[8,25]$

$$
D=2 / \sigma+1
$$

in order to establish a relationship between the $\epsilon$-expansion result and ours. The $\sigma \leftrightarrow D$ relation has been pursued extensively, and more refined formulas were proposed. The above expression (11) has an advantage in that it is given in a closed form.

The overall features of our data and the analytic result (10) resemble to each other. Our simulation result suggests that a slight enhancement as to the analytic formula for the large- $\sigma$ regime, $\sigma \gtrsim 1$. Remarkably enough, the above formula (11) up to $\mathrm{O}\left(\epsilon^{1}\right)$ may be practically of use to establish a relationship between the power of algebraic decay $\sigma$ and the low-lying excitation spectrum.

The Higgs mass $m_{H}$ may have an uncertainty, because it has a finite life time; the reciprocal life time corresponds to the intrinsic $m_{H}$-peak width. According to the $\epsilon$-expansion analysis [35], the scaled peak width $\delta m_{H} / \Delta$ should also take a universal constant

$$
\frac{\delta m_{H}}{\Delta}=\frac{\sqrt{2} \pi \epsilon}{40}
$$

At $\sigma=1(D=3)$, this formula yields the scaled peak width $\delta m_{H} / \Delta=$ $0.11 \ldots$ Taking this intrinsic uncertainty into account, our result and the analytical formula (10) do not display substantial disagreement for the small$\sigma$ side, $\sigma \lesssim 1$, As mentioned in Sec. 2.2 , the series of data $m_{H} / \Delta$ terminate around $\sigma \approx 1.6(D \approx 2.25)$. Such a feature is supported by the analytic formula (12) in the sense that for large $\sigma$ (large $\epsilon$ ), the Higgs particle becomes obscure nonetheless.

We make a brief overview for the fixed- $D=3(=2+1)$-lattice analyses. By means of the quantum Monte Carlo method, the estimates, $m_{H} / \Delta=$ 
2.1(3) [36, 37] and 3.3(8) [38], were reported, whereas the exact diagonalization method yields 2.1(2) [39]. With the functional-renormalization-group method, the results, $m_{H} / \Delta=2.4$ [40] and 2.2 [41], were obtained, while the $\epsilon$-expansion method [35] yields 1.67. The present simulation admits $m_{H} / \Delta=1.78(11)$ at $\sigma=1(D=3)$. These results seem to be rather scattered. At present, it is not clear whether the discrepancy should be attributed to the above-mentioned intrinsic uncertainty (12), or a systematic one. Actually, the analyticity for the dynamical scalar susceptibility $\chi_{s}(\omega)$ [35] reveals an appreciable discrepancy between the $m_{H}$-pole position and the actual peak position; from the latter, the quantum Monte Carlo results were read off. Clearly, detailed information as to the incoherent part of $\chi_{s}(\omega)$ would be desirable so as to resolve the peak position out of the incoherent background. To the best of author's knowledge, neither the $m_{H}$-peak shape nor the life time has been investigated quantitatively. At present, the analytic result (10) and ours would be "easier to reconcile with the lower of" [35] those preceding results [36, 37, 38, 39, 40, 41].

\section{Summary and discussions}

The quantum $X Y$ spin chain (2) with the algebraically decaying interactions, $1 / r^{1+\sigma}$, was investigated by means of the exact diagonalization method. This method enables us to identify the spectral gaps, $m_{H}$ and $\Delta$, by specifying the quantum numbers to Eqs. (3) and (4), respectively. As a preliminary survey, we analyzed the finite-size-scaling behavior [25] for $m_{H}$, and as a byproduct, we obtained the $\sigma$-dependent dynamical critical exponent $z$. The result $z$ seems to obey the approximate formula (7), suggesting that the formula may be practically of use for a wide range of $\sigma$; the discrepancies around both upper and lower critical thresholds, $\sigma=2$ and $2 / 3$, respectively, should be attributed to the notorious corrections to finite-size scaling $[3,10,11,26]$.

We then turn to the analysis of the critical amplitude ratio $m_{H} / \Delta$. Our data obey the $\epsilon$-expansion result, Eq. (10), for the small- $\sigma$ side, $\sigma \lesssim 1$, whereas the simulation result displays a slight enhancement as to the analytical formula for large $\sigma$. It has to be mentioned that there should exist an intrinsic uncertainty, Eq. (12), as to $m_{H} / \Delta$ due to the finite life time for $m_{H}$. Taking this uncertainty into account, the overlap between the analytical formula (10) and the numerical result do not exhibit substantial disagreement in the small- $\sigma$ side, $\sigma \lesssim 1$. For exceedingly large $\sigma$, the Higgs particle gets unstable, and the amplitude ratio $m_{H} / \Delta$ becomes obscure nonetheless. Note 
Figure 1: The universality chart $[25,30,31,32,33,34]$ for the quantum $X Y$ spin chain with the interactions decaying as a power law $1 / r^{1+\sigma}$ of the distance between spins $r$ is presented. For $\sigma \gtrsim 2$, the long-range interaction becomes irrelevant, and the universality class of the order-disorder phase transition falls into that of the two-dimensional $(D=$ 2) classical $X Y$ model, whereas for $\sigma>2 / 3$, the system exhibits the mean-field $(D=$ 4) criticality. In the intermediate regime $2 / 3 \leq \sigma \lesssim 2$, the criticality with fractional effective dimensionality $2 \leq D \leq 4$ is realized. That is, the power of the algebraic decay $\sigma$ interpolates smoothly the upper and lower critical dimensions. At these boundaries, there arise logarithmic corrections $[3,10,11,26]$ to finite-size scaling.

that both gaps $m_{H}$ and $\Delta$ are experimentally observable [45] in proximity to the critical point. It would be intriguing that the amplitude ratio $m_{H} / \Delta$ reflects the power of algebraic decay $\sigma$ as a monotonic function; actually, the correlation-length critical exponent $\nu$ displays a non-monotonic behavior [11] as to the variation of $\sigma$.

As for the fixed- $D=3(=2+1)$ lattice simulation, the results, $m_{H} / \Delta=$ 2.1(3) $[36,37]$ and 3.3(8) [38], were reported with the quantum Monte Carlo method, whereas the exact diagonalization method yields 2.1(2) [39]. Through the functional-renormalization-group method, the estimates, $m_{H} / \Delta=2.4$ [40] and 2.2 [41], were obtained, while the $\epsilon$-expansion method yields 1.67 . The present simulation admits $m_{H} / \Delta=1.78(11)$ at $\sigma=1(D=3)$. These results seem to be rather scattered. At present, it is not clear whether the disagreement should be attributed to the above-mentioned intrinsic uncertainty or a systematic one. Actually, the analyticity for the dynamical scalar susceptibility $\chi_{s}(\omega)[35]$ reveals an appreciable discrepancy between the $m_{H}$-pole position and the actual spectral-peak position; from the latter, the quantum Monte Carlo results were read off. Clearly, detailed information as to the incoherent part of $\chi_{s}(\omega)$ would be desirable so as to resolve $m_{H}$ out of the background. This problem is left for the future study.

\section{References}

[1] M. E. Fisher, S.-k. Ma, and B. G. Nickel, Phys. Rev. Lett. 29 (1972) 917. 
Figure 2: A schematic drawing of the low-lying spectrum for the quantum long-range $X Y$ spin model (2) with the $X Y$ interaction $J$ is presented. In the ordered phase $J>J_{c}$, there opens the Higgs gap $m_{H}$ embedded within the Goldstone-excitation continuum, whereas in the disordered phase $J<J_{c}$, the paramagnetic gap $\Delta$ appears. The scaling behavior for $m_{H}$ as well as the critical amplitude ratio $m_{H}(J) /\left.\Delta\left(2 J_{c}-J\right)\right|_{J \rightarrow J_{c}^{+}}$are the main concern. 


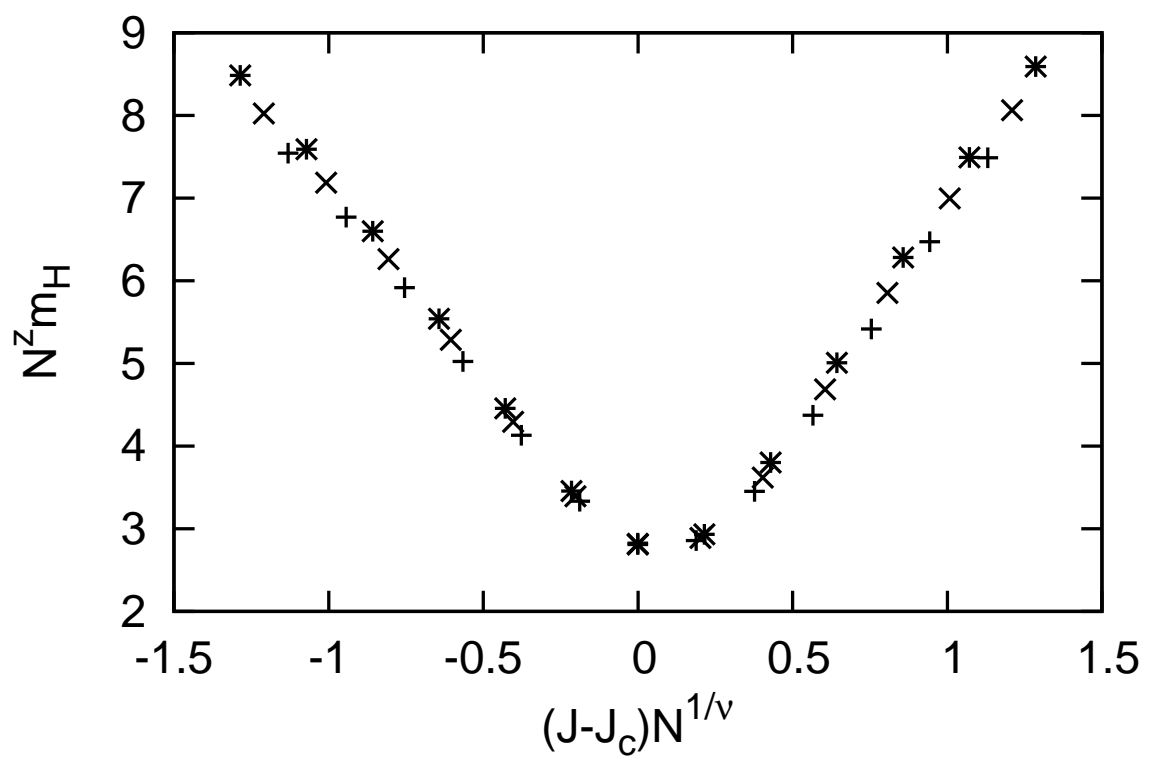

Figure 3: The scaling plot, $\left(J-J_{c}\right) N^{1 / \nu}-N^{z} m_{H}$, is presented for $\sigma=1.2$, and the system sizes, $(+) N=18,(\times) 20$, and $(*) 22$, with the scaling parameters, $J_{c}=0.30785, z=0.594$ and, $1 / \nu=0.636$. The Higgs gap opens in the ordered phase $J>J_{c}$. This mode seems to be massive in the adjacent phase $J<J_{c}$ as well, supporting the preceding observations with the quantum Monte Carlo method [37, 38]. 


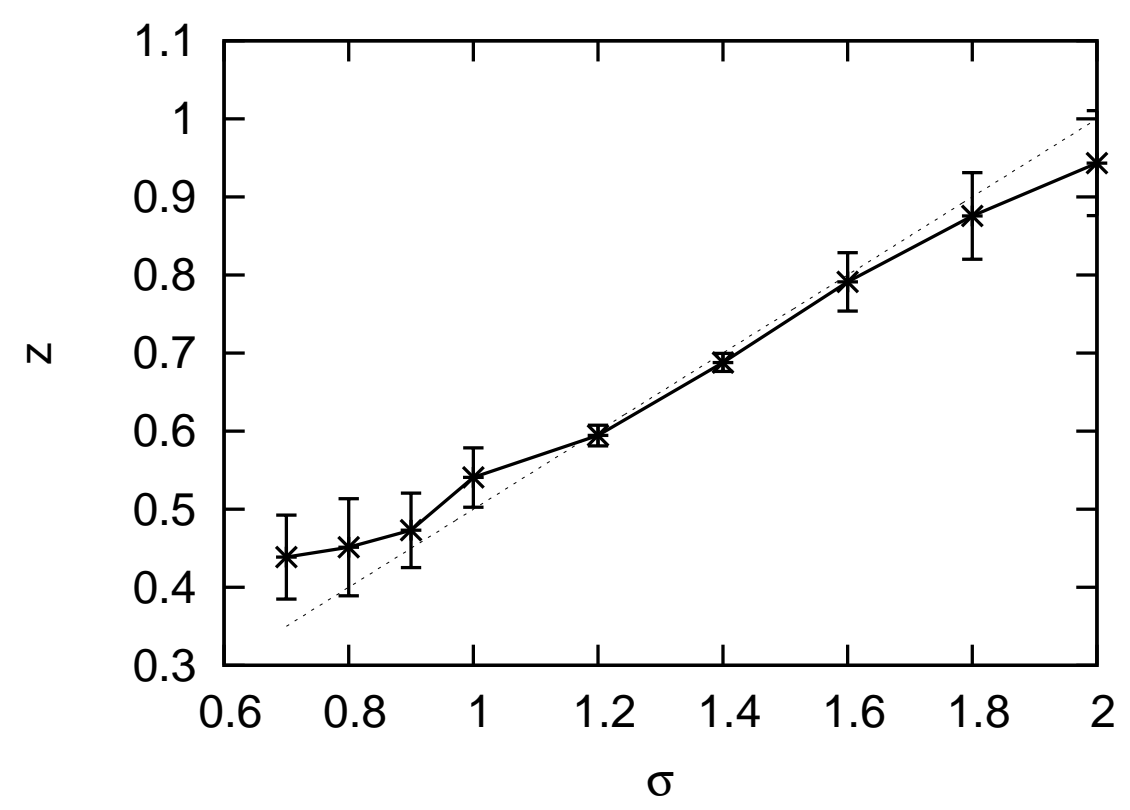

Figure 4: The dynamical critical exponent $z$ is presented for the power of algebraic decay $\sigma$. An approximate formula [25], Eq. (7), is presented (dotted) as well. This formula is validated at least for small $\sigma$. Deviations around the lower and upper critical thresholds, $\sigma=2 / 3$ and 2 , respectively, may be attributed to the notorious logarithmic corrections to finite-size scaling $[3,10,11,26]$. 


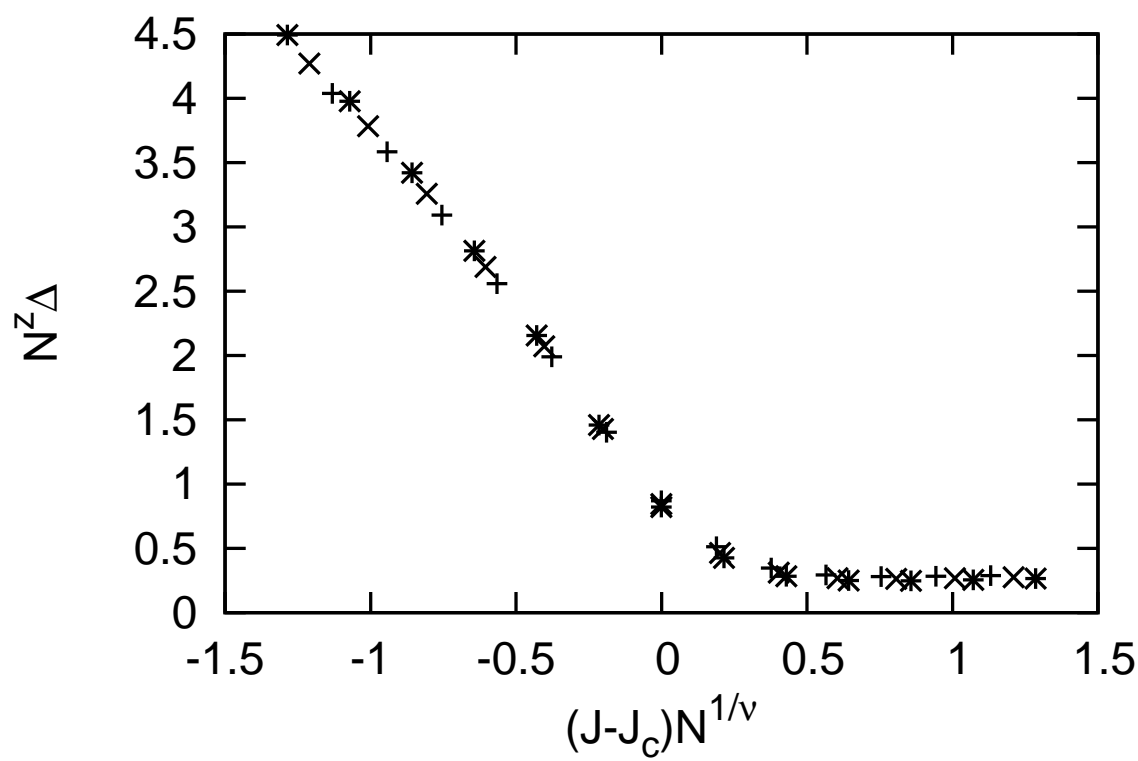

Figure 5: The scaling plot, $\left(J-J_{c}\right) N^{1 / \nu}-N^{z} \Delta$, is presented for $\sigma=1.2$, and the system sizes, $(+) N=18,(\times) 20$, and $(*) 22$. The scaling parameters, $J_{c}=0.30785, z=0.594$ and $1 / \nu=0.636$, are the same as those of Fig. 3. The paramagnetic gap $\Delta$ opens in the disordered phase $J<J_{c}$. This branch becomes the Goldstone gapless mode in the adjacent phase $J>J_{c}$, forming the Goldstone continuum in the low-energy spectrum. 


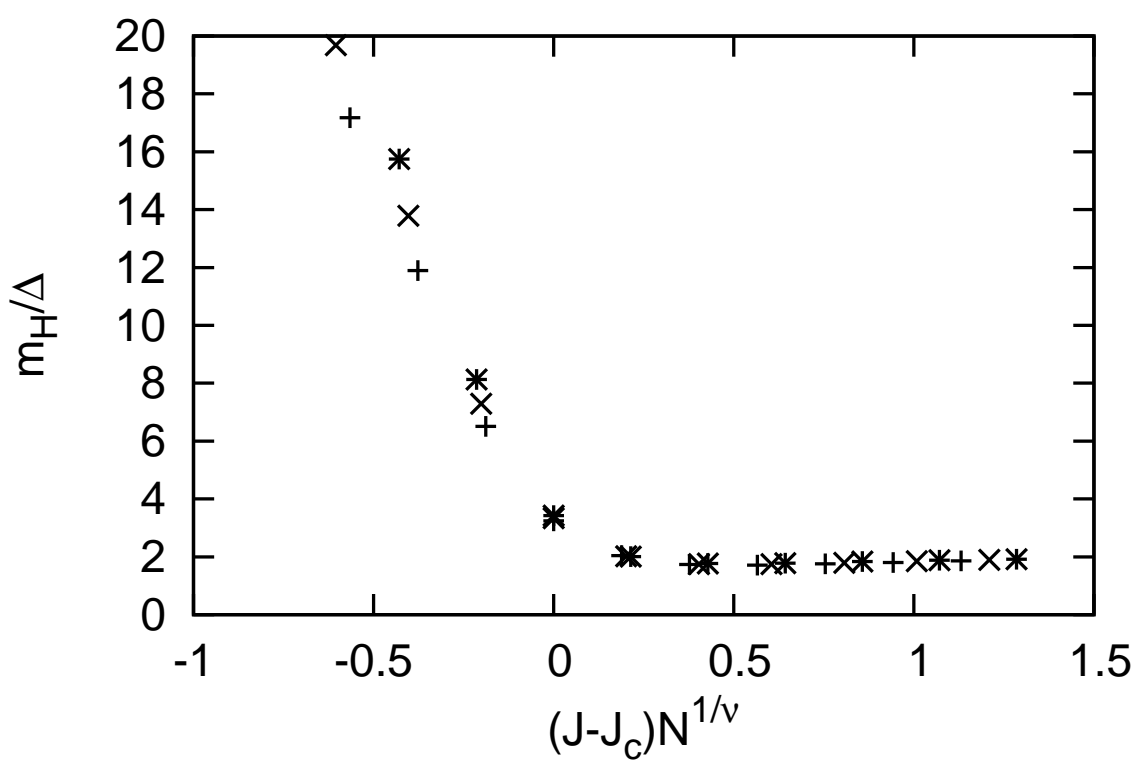

Figure 6: The scaling plot, $\left(J-J_{c}\right) N^{1 / \nu}-m_{H}(J) / \Delta\left(2 J_{c}-J\right)$, is presented for $\sigma=1.2$, and the system sizes, $(+) N=18,(\times) 20$, and $(*) 22$. The scaling parameters, $J_{c}=$ 0.30785 , and $1 / \nu=0.636$, are the same as those of Fig. 3. In the ordered phase $J>J_{c}$, there appears a plateau with the height $m_{H} / \Delta \approx 1.8$, which admits an estimate for the amplitude ratio; see text for details. 


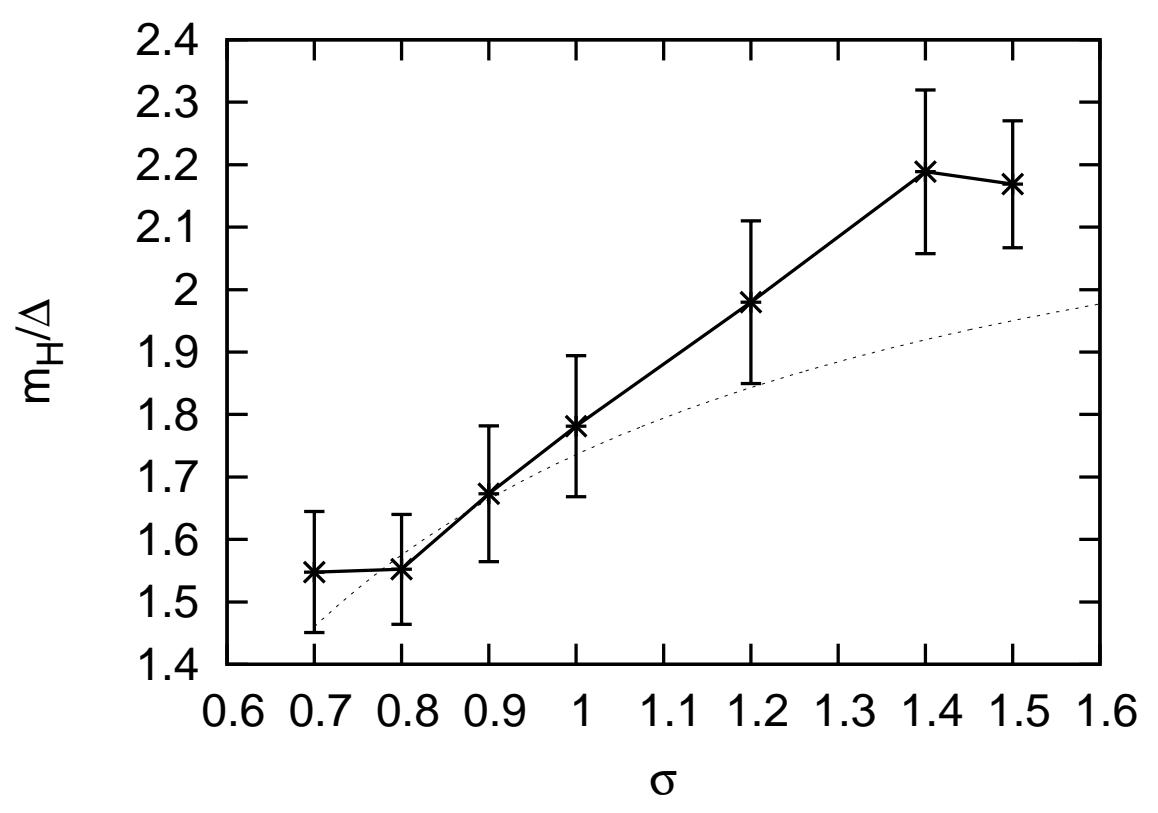

Figure 7: The critical amplitude ratio $m_{H} / \Delta$, Eq. (1), is presented for various $\sigma$. The series of solutions terminate around $\sigma \approx 1.6$, because the Higgs excitation becomes unstable for exceedingly large $\sigma \gtrsim 1.6$. According to the formula (11), the power $\sigma \approx 1.6$ corresponds to $D \approx 2.25$, which is about to reach the lower critical dimension $D=2$. As a reference, the $\epsilon(=4-D)$-expansion formula [35], Eq. (12), is shown as a dotted curve. 
[2] J. Sak, Phys. Rev. B 8 (1973) 281.

[3] E. Luijten and H. W. J. Blöte, Phys. Rev. Lett. 89 (2002) 025703.

[4] M. Picco, arXiv:1207.1018.

[5] T. Blanchard, M. Picco, and M. A. Rajabpour, Europhys. Lett. 101 (2013) 56003.

[6] P. Grassberger, J. Stat. Phys. 153 (2013) 289.

[7] G. Gori, M. Michelangeli, N. Defenu, and A. Trombettoni Phys. Rev. E 96 (2017) 012108.

[8] M. C. Angelini, G. Parisi, and F. Ricchi-Tersenghi, Phys. Rev. E 89 (2014) 062120.

[9] J. S. Joyce, Phys. Rev. 146 (1966) 349.

[10] E. Brezin, G. Parisi, and F. Ricci-Tersenghi, J. Stat. Phys. 157 (2014) 855 .

[11] N. Defenu, A. Trombettoni, and A. Codello, Phys Rev. E 92 (2015) 052113.

[12] N. Defenu, A. Trombettoni, and S. Ruffo, Phys. Rev. B 94 (2016) 224411.

[13] R. Goll and P. Kopietz, Phys. Rev. E 98 (2018) 022135.

[14] E. Flores-Sola, M. Weigel, R. Kenna, and B. Berche, Eur. Phys. J. Special Topics 226 (2017) 581.

[15] T. Horita, H. Suwa, and S. Todo, Phys. Rev. E 95 (2017) 012143.

[16] G. Sun, Phys. Rev. A 96 (2017) 043621.

[17] W. Wu, B. Ellman, T. F. Rosenbaum, G. Aeppli, and D. H. Reich, Phys. Rev. Lett. 67 (1991) 2076.

[18] J. W. Britton, B. C. Sawyer, A. C. Keith, C.-C. Joseph Wang, J. K. Freericks, H. Uys, M. J. Biercuk, J. J. Bollinger, Nature 484 (2012) 489. 
[19] R. Islam, C. Senko, W.C. Cambell, S. Korenblit, J. Smith, A. Lee, E. E. Edwards, C.-C. J. Wang, J. K. Freericks, and C. Monroe, Science 340 (2013) 583.

[20] P. Richerme, Z.-X. Gong, A. Lee, C. Senko, J. Smith, M. Foss-Feig, S. Michalakis, A.V. Gorshkov, and C. Monroe, Nature 511 (2014) 198.

[21] P. Jurcevic, B.P. Lanyon, P. Hauke, C. Hempel, P. Zoller, R. Blatt, and C.F. Roos, Nature 511 (2014) 202.

[22] A. de Paz, A. Sharma, A. Chotia, E. Maréchal, J.H. Huckans, P. Pedri, L. Santos, O. Gorceix, L. Vernac, and B. Laburthe-Tolra, Phys. Rev. Lett. 111 (2013) 185305.

[23] N. Laflorencie, I. Affleck, and M. Berciu, J. Stat. Mech.: Theory and Experiment, P12001 (2005).

[24] A. Dutta and J. K. Bhattacharjee, Phys. Rev. B 64 (2001) 184106.

[25] N. Defenu, A. Trombettoni, and S. Ruffo, Phys. Rev. B 96 (2017) 104432 .

[26] S. Fey and K. P. Schmidt, Phys. Rev. B 94 (2016) 075156.

[27] A. W. Sandvik, Phys. Rev. Lett. 104 (2010) 137204.

[28] T. Koffel, M. Lewenstein, and L. Tagliacozzo, Phys. Rev. Lett. 109 (2012) 267203.

[29] S. Humeniuk, Phys. Rev. B 93 (2016) 104412.

[30] L. S. Campana, L. De Cesare, U. Esposito, M. T. Mercaldo, and I. Rabuffo, Phys. Rev. B 82 (2010) 024409.

[31] Z.-X. Gong, M. F. Maghrebi, A. Hu, M. Foss-Feig, P. Richerme, C. Monroe, and A. V. Gorshkov, Phys. Rev. B 93 (2016) 205115.

[32] M. F. Maghrebi, Z.-X. Gong, and A. V. Gorshkov, Phys. Rev. Lett. 119 (2017) 023001.

[33] I. Frérot, P. Naldest, and T. Roscilde, Phys. Rev. B 95 (2017) 245111.

[34] S. S. Roy and H. S. Dhar, arXiv:1809.02335. 
[35] Y. T. Katan and D. Podolsky, Phys. Rev. B 91 (2015) 075132.

[36] S. Gazit, D. Podolsky, and A. Auerbach, Phys. Rev. Lett. 110 (2013) 140401.

[37] S. Gazit, D. Podolsky, A. Auerbach, and D. P. Arovas, Phys. Rev. B 88 (2013) 235108.

[38] K. Chen, L. Liu, Y. Deng, L. Pollet, and N. Prokof'ev, Phys. Rev. Lett. 110 (2013) 170403.

[39] Y. Nishiyama, Nucl. Phys. B 897 (2015) 555.

[40] A. Rançon and N. Dupuis, Phys. Rev. B 89 (2014) 180501.

[41] F. Rose, F. Léonard and N. Dupuis, Phys. Rev. B 91 (2015) 224501.

[42] N. Dupuis, Phys. Rev. E 83 (2011) 031120.

[43] D. Podolsky, A. Auerbach, and D. P. Arovas, Phys. Rev. B 84 (2011) 174522 .

[44] D. Pekker and C.M. Varma, Annual Rev. Condens. Matter Phys. 6 (2015) 269.

[45] M. Endres, T. Fukuhara, D. Pekker, M. Cheneau, P. Schauß, C. Gross, E. Demler, S. Kuhrand and I. Bloch, Nature 487 (2012) 454.

[46] V. G. Rousseau, R. T. Scalettar, and G. G. Batrouni, Phys. Rev. B 72 (2005) 054524.

[47] A. W. Sandvik, S. Daul, R. R. P. Singh, and D. J. Scalapino, Phys. Rev. Lett. 89 (2002) 247201.

[48] J. D'Emidio and R. K. Kaul, Phys. Rev. B 93 (2016) 054406.

[49] Y. Deng and H.W.J. Blöte, Phys. Rev. E 68, 036125 (2003).

[50] R. Botet, R. Jullien, and M. Kolb, Phys. Rev. B 28 (1983) 3914.

[51] U. Glaus and T. Schneider, Phys. Rev. B 30 (1984) 215.

[52] J. Sólyom and T. A. L. Ziman, Phys. Rev. B 30 (1984) 3980. 
[53] I. Homrighausen, N. O. Abeling, V. Zauner-Stauber, and J. C. Halimeh, Phys. Rev. B 96 (2017) 104436.

[54] L. Vanderstraeten, M. Van Damme, H. P. Büchler, and F. Verstraete, Phys. Rev. Lett. 121 (2018) 090603.

[55] I. Frérot, P Naldesi, and T. Roscilde, Phys. Rev. Lett. 120 (2018) 050401.

[56] F. Rose, F. Benitez, F. Léonard, and B. Delamotte, Phys. Rev. D 93 (2016) 125018. 